Draft version September 17, 2018

Preprint typeset using $\mathrm{L}_{\mathrm{A}}^{\mathrm{A}} \mathrm{E} \mathrm{X}$ style emulateapj v. 5/2/11

\title{
INTERNAL GRAVITY WAVES MODULATE THE APPARENT MISALIGNMENT OF EXOPLANETS AROUND HOT STARS
}

\author{
T.M. ROGERS \\ Department of Planetary Sciences, University of Arizona, Tucson, AZ, 85719
}

D.N.C. LIN

Astronomy and Astrophysics Department, University of California, Santa Cruz, CA 95064 and Kavli Institute for Astronomy and Astrophysics and School of Physics, Peking University, China

H.H.B. LAU

Argelander-Institut for Astronomie Universit Bonn Auf dem Huegel 7153121 Bonn Germany and Monash Centre for Astrophysics, School of Mathematical Sciences, Monash University, Australia Draft version September 17, 2018

\begin{abstract}
We propose that the observed misalignment between extra-solar planets and their hot host stars can be explained by angular momentum transport within the host star. Observations have shown that this misalignment is preferentially around hot stars, which have convective cores and extended radiative envelopes. This situation is amenable to substantial angular momentum transport by internal gravity waves (IGW) generated at the convective-radiative interface. Here we present numerical simulations of this process and show that IGW can modulate the surface rotation of the star. With these twodimensional simulations we show that IGW could explain the retrograde orbits observed in systems such as HAT-P-6 and HAT-P-7, however, extension to high obliquity objects will await future threedimensional simulations. We note that these results also imply that individual massive stars should show temporal variations in their $\mathrm{v}$ sini measurements.

Subject headings: internal gravity waves, angular momentum redistribution, extra-solar planets, hot jupiters
\end{abstract}

\section{INTRODUCTION}

Jupiter-mass planets with a few day periods (hereafter hot Jupiters) are found around 1-2\% of solar type stars. At their present-day location $(<0.05 \mathrm{AU})$, in situ formation through core accretion (Pollack et al. 1996) is challenging because a1) their natal disks are relatively hot $\left(>10^{3} \mathrm{~K}\right)$, a2) the local Keplerian velocity is $>10^{2}$ $\mathrm{km} \mathrm{s}^{-1}$ (ie much larger than the surface escape speed of any known planets), and a3) the tidal perturbation of their host stars is strong. Consequently, b1) only a modest amount of refractory grains can avoid evaporation in this region, b2) high-speed collisions among planetesimals generally lead to their disruption, b3) the asymptotic mass of protoplanetary embryos is limited, and b4) the gas accretion is quenched.

These issues have led to a paradigm shift that hot Jupiters may have relocated over large distances from the preferred sites of their assemblage (Ida \& Lin 2004, 2010). The physical cause of planetary relocation remains a controversial issue. A widely adopted "disk-migration" scenario attributes the cause of hot Jupiters' orbital evolution to their tidal interaction with their natal disks (Lin et al. 1996). Their orbital decay is stalled when they enter into a central cavity induced by the host stars' magnetosphere (Koenigl 1991) or when their natal disk is severely depleted. This disk-migration scenario natu-

tami@lpl.arizona.edu

İin@ucolick.org

ṅbiau@astro.uni-bonn.de rally accounts for the origin of multiple planets which are locked in mean motion resonances (Lee \& Peale 2002)

Distant gas giants may also venture close to their host stars if their eccentricity is near unity through dynamical excitation by the Kozai (1962) resonance (Wu \& Murray 2003; Nagasawa \& Ida 2011), planet-planet scattering (Chatterjee et al. 2008), or secular chaos (Wu \& Lithwick 2011). Tidal dissipation within the scattered planets would circularize their orbits if their periastron distance can reach within a few times their Roche radii (Rasio \& Ford 1996). However, mass loss during the extremely close encounters also leads to either escape (Guillochon et al. 2011) or residual cores as short-period super Earths (Liu et al. 2012). This dynamical scenario predicts a pile up in the period distribution which does not seem to match that observed with a control sample (Howard et al. 2010).

Recent observations of the Rossiter-McLaughlin (RM) effect (Ohta et al. 2005) indicate that the spin of many relatively massive and hot $(>6,300 \mathrm{~K})$ main sequence stars is misaligned with their planets' angular momentum vector (Winn et al. 2010, Schlaufman 2010). This misalignment is an expected outcome of the dynamical scenario (Fabrycky \& Tremaine 2007), although its correlation with the stellar mass requires assumptions on the poorly know tidal dissipation mechanism inside main sequence stars (Winn et al. 2011, Lai 2012).

The observed spin-orbit misalignment would pose a challenge to the disk-migration scenario for the origin of hot Jupiters (Triaud et al. 2010, Winn et al. 2011) 
if the host stars retain the angular momentum they accreted from the protostellar disks, out of which the planets formed. Within the observational uncertainty, this assumption is consistent with that observed in Kepler 30 (Sanchis-Ojeda et al. 2012), but it cannot account for the 7 degree tilt in the Sun's spin axis with respect to the angular momentum vector of the planetary orbits.

Stars generally form in a turbulent cloud complex where fragments undergo chaotic encounters as they collapse to form stars through disk accretion. The spin orientation of protostellar disks may re-orient episodically, especially during the disk formation. Bate (2010) suggested that this process may have led to the observed misalignment between planets' orbital angular momentum vector and the spin axis of their host stars as well as differential rotation. But this possibility may depend on whether 1) there is adequate mass in the disk to form planets, 2) there is sufficient residual gas to re-orient planets' orbital plane during disk depletion, and 3) there is enough coupling between the stars and their surrounding disks to realign the spin of their outer envelope.

In this paper, we consider another possibility: that differential spin may naturally arise in relatively massive and hot stars even in the limit that they started with uniform spin. We show that internal gravity waves (IGW) generated at the interface between the convective core and radiative envelope transport angular momentum outward. These waves dissipate in a shell near the surface of the star forcing the surface (including the photosphere) to rotate differently than the bulk of the star and to vary in time.

\section{RESULTS}

\subsection{Model Setup}

In order to study the angular momentum transport by waves in a massive star we solve the full set of hydrodynamic equations in the anelastic approximation. These equations are solved in two-dimensional cylindrical coordinates representing an equatorial slice of the star. A complete description of the equations and numerical method can be found in Rogers \& Glatzmaier (2005). The reference state model is that of a $3 M_{\odot}$ star and is calculated using the Cambridge stellar evolutionary code STARS (Eggleton 1971) 1 In this model the convective core occupies the inner $\approx 14 \%$ by radius and the radiative envelope extends to the stellar surface at $\approx 1.6 R \odot^{2}$. Over the simulated domain, the density varies from $40 \mathrm{gm} / \mathrm{cm}^{3}$ in the core to $10^{-3} \mathrm{gm} / \mathrm{cm}^{3}$ at the surface. Here we report mainly on the results of a model in which the convecting core is initially rotating 1.5 times faster than the radiative envelope and the average rotation is approximately ten times solar, designated MDR. An additional model with no initial rotation, designated MUR, will be briefly discussed.

\subsection{Angular Momentum Transport by Internal Gravity Waves}

\footnotetext{
1 Although we use a $3 M_{\odot}$ model here we expect the results to be qualitatively representative of any star with core convection and an extended radiative envelope.

2 We do not in this first attempt include a surface convection zone for numerical efficiency.
}

In Figure 1 (a-e) we show the vorticity at five different times during the simulation for MDR. We can immediately see that the small convecting region efficiently generates IGW ${ }^{3}$ which propagate into the radiative envelope with amplitudes similar to that of convection (wave velocity amplitudes at generation are $\approx 10^{3}-10^{5} \mathrm{~cm} / \mathrm{s}$ ). The wave generation process is extremely time and location dependent. In Figure 1a one can clearly see that waves are generated where an upwelling plume hits the convective-radiative interface. At one time waves are generated at one main location, at another a different location and at another at multiple locations (see Figures $1 \mathrm{~b}$ and $1 \mathrm{c}$ as well). Therefore, the wave pattern seen in the radiative envelope is some combination of waves generated at various times and locations. One can also see in Figure 1a that the wave patterns are predominantly horizontal or concentric, reflecting the fact that these waves propagate mostly horizontally because of the smallness of $\omega / N$, where $\omega$ is the wave frequency and $\mathrm{N}$ is the Brunt-Vaisala frequency. The wave propagation is therefore, a spiral propagation outward.

Once generated, the propagation and dissipation of the waves depends sensitively on the properties of the waves. Low frequency waves are generated with higher amplitudes than high frequency waves (the generation spectrum we measure is $\left.A(\omega) \propto e^{\left(b_{1} \omega+b_{2} \omega^{2}\right)}\right)$. However, on their propagation outward low frequency waves are dissipated by radiative diffusion more strongly than their high frequency counterparts. The radiative diffusion acts nearly as an equalizer, so that while high and low frequency waves are generated with different amplitudes, once they reach the surface there is less dispersion. The waves that reach the surface with the highest amplitude are those waves with high enough frequency to escape substantial radiative diffusion but low enough frequency to be generated efficiently, these tend to be waves with frequencies $\approx 10 \mu \mathrm{Hz}$ and large scales (horizontal wavenumber, $\mathrm{k}, 1-3)$. It is these mid-frequency range waves that contribute the most to the angular momentum transport at the stellar surface. Waves with this combination of frequency and wavenumber reach the surface with velocities approaching $\approx 10^{5} \mathrm{~cm} / \mathrm{s}$, have Froud $\AA^{4}$ numbers close to 1 and are therefore, nonlinear. Some evidence of wave breaking, rippled surfaces and large amplitudes, can be seen in figure $1 \mathrm{~b}$. While we see some evidence of wave breaking, the actual process by which waves initially dissipate in the outer layers is not fully understood. Several physical processes could contribute including: 1) nonlinear wave breaking 2) wavewave interaction as waves reflect off the top boundary or 3) wave-wave interactions as waves internally reflect when propagating into a region of rapidly varying $\mathrm{N}$.

Because of the initially imposed differential rotation at the convective-radiative interface, prograde waves are doppler-shifted to higher frequencies, while retrograde waves are shifted to lower frequencies. Therefore, radiative diffusion, being frequency dependent (the radiative damping length is proportional to $\left.\omega^{4}\right)$, dissipates

\footnotetext{
3 We use the term "wave" here loosely to mean "disturbance".

4 The Froude number is a general measure of wave linearity and is defined as $w^{\prime} / L N$, where w' is a velocity amplitude, $\mathrm{L}$ is the wavelength and $\mathrm{N}$ is the Brunt-Vaisala frequency. Numbers close to unity indicate nonlinearity.
} 
retrograde waves slightly more than prograde waves. Consequently, upon arrival at the surface, retrograde waves have slightly lower amplitude than their prograde counter-parts and therefore, contribute slightly less to the angular momentum evolution. This means that, although both prograde and retrograde waves reach the surface and dissipate, over time, prograde waves deposit more angular momentum and the region accelerates.

Eventually the shear flow reaches an amplitude such that it represents a critical layer to many of the dominant phase speed waves (Booker \& Bretherton 1967). A critical layer is defined as the position where the local angular velocity is equal to the horizontal phase speed of the wave, $c_{p h}$. In the ray-tracing description of IGW the vertical wavenumber goes to infinity at a critical layer, therefore, the time to approach the critical layer goes to infinity 5 This means that any small dissipation leads to complete absorption of the wave at the critical layer. For example, at the position labeled (1) in Figure $2 \mathrm{~b}$, the angular velocity is $\approx 10^{-5} \mathrm{~Hz}$, therefore, a wave with frequency $10 \mu \mathrm{Hz}$ and horizontal wavenumber one, has a horizontal phase speed $10^{-5} \mathrm{~Hz}$ and experiences critical layer absorption. Indeed, any wave in which $c_{p h}=\omega / k \geq 10^{-5} \mathrm{~Hz}$ will encounter the critical layer and deposit most, if not all, of its angular momentum locally. This causes the rapid increase in the angular velocity seen between times labeled (1) and (2) in Figure $2 \mathrm{~b}$.

To quantify the efficiency of angular momentum transport by IGW we turn to the evolution equation for the mean angular velocity:

$$
\frac{\partial \bar{U}}{\partial t}=-\frac{1}{\rho r} \frac{\partial r\left(\rho \overline{u_{r} u_{\phi}}\right)}{\partial r}+\nu\left(\frac{\partial^{2} \bar{U}}{\partial r^{2}}+\left(\frac{2}{r}+h_{\rho}\right) \frac{\partial \bar{U}}{\partial r}\right)
$$

the first term on the right hand side (rhs) of (1) represents the angular momentum transport by IGW or the wave momentum flux (the second term on the rhs of (1) represents viscous dissipation, near the surface the viscous dissipation is always at least two orders of magnitude smaller than the momentum flux by waves). This value is $\approx 10 \mathrm{~cm} / \mathrm{s}^{2}$ at the base of the radiative envelope and around $1 \mathrm{~cm} / \mathrm{s}^{2}$ at the surface, although there are order 10-100 variations in time. This can lead to rapid time variation of the mean flow. The timescale for the prograde layer to grow to its peak value is extremely rapid. Within about 9 rotation periods, or approximately $10^{-10} \tau_{E S}$, where $\tau_{E S}$ is the Eddington-Sweet time, the angular velocity at the surface has increased by a factor of 10 . Stated another way, in the rotating frame of reference the linear velocity at the surface has increased from zero to $\approx 10^{6} \mathrm{~cm} / \mathrm{s}$ in 9 rotation periods, consistent with the values of momentum flux given above.

As time progresses, the prograde layer migrates as continuously generated waves steepen and dissipate within the critical layer. This causes the prograde layer to propagate toward the convection zone in time (Figure

\footnotetext{
5 The critical level is defined as the place where the horizontal phase speed of the wave is equivalent to the mean flow speed. As the wave is incident on the critical level there is a region below the critical level where substantial wave dissipation occurs, which is referred to as the citical layer. We have not necessarily adhered to these subtle distinctions in our physical description.
}

2a), similar to what occurs in the Quasi-Biennial oscillation (Baldwin et al. 2001) in our own atmosphere and to the Plumb \& McEwan laboratory experiment (Plumb \& McEwan 1978). Eventually the entire radiative envelope is spun up. However, because of angular momentum conservation, the convection zone slows down (see Figure $2 \mathrm{a}$ ). This produces a differential rotation profile that favors retrograde waves at the surface, so the process reverses and eventually, the surface will spin retrograde. We have not been able to run this model long enough for the reversal to occur, however, one can see the slow decay of the prograde shear in Figure $2 \mathrm{~b}$ as well as retrograde pockets in Figure 1e. At the current rate of deceleration (which is substantially slower than the rate of acceleration since no retrograde critical layer has yet formed) the prograde radiative envelope would take approximately a few hundred rotation periods to reverse, or $10^{-7} \tau_{E S}$, which astronomically speaking, is still an extremely short timescale. The growth and reversal timescales are inversely proportional to the wave momentum flux (Plumb 1977). The reversal timescale is longer than the initial growth time because the convection zone contains most of the mass, therefore, when it slows down the differential rotation between it and the surrounding radiation zone is weaker than the initially imposed differential rotation. Hence, the amplitude variation between prograde and retrograde waves at the surface is reduced and the net momentum flux is lower.

In Figure 1(f-j) we show the vorticity for the model MUR, in which there is no initial rotation. Here we clearly see dynamics similar to that seen in Figure (a-e) but the initial surface shear that develops in this model is retrograde. In a model with uniform initial rotation the sense of the initial surface shear layer is random as there is no preferred direction. Otherwise, this model evolves similarly to the one previously described with the development of a critical layer, rapid momentum transport and momentum fluxes similar to those quoted above for MDR. Although we have only presented these two models we note that this is a very robust mechanism as a surface shear flow has developed in all models we have run, whether initially uniformly or differentially rotating. The amplitude of the shear and its growth rate depend on the model details but the time dependent shear development is rigorous. The two models presented demonstrate the large variation in amplitude and sign that surface flows on hot stars can experience.

\section{DISCUSSION}

In the above models we have shown that IGW can efficiently transport angular momentum in stars with convective cores and extended radiative envelopes. This transport causes the angular velocity profile to vary substantially in radius and time. In the model presented the angular velocity at the surface changed by a factor of 10 in a timescale minuscule compared to other relevant timescales. In all cases run the angular velocity changes in time. This has significant consequences for observations of massive star rotation, as these models indicate that the rotation of the surface layers is not necessarily representative of the rotation of the entire star. They also indicate that the surface rotation of the star is time dependent. This is a robust process, which happens in our own atmosphere (Baldwin et al. 2001), has been 
demonstrated experimentally (Plumb \& McEwan 1978), and shown here numerically. It likely occurs in any star with a convective core and extended radiative envelope and has many interesting consequences.

This mechanism provides a natural explanation for why mis-aligned planetary systems tend to be around hot stars. In hot stars which have convective cores and radiative envelopes IGW transport sufficient angular momentum to cause the surface of the star to rotate differently than it did when the planetary system was formed. Although our 2D models only strictly explain retrograde systems we view this work as a proof of concept and application to other high-obliquity objects will await future 3D simulations ${ }^{6}$ These simulations imply, at the very least, that the equatorial angular velocity at the surface could be time-dependent as the IGW-driven shear flow varies in time. This physical effect would lead to variations in measured RM effect. Multiple observations of the exoplanet system XO-3 by Hébrard et al. (2008), Winn et al. (2009) and Hirano et al. (2011) reveal an unexplained variation in spin-misalignment, which could be interpreted as a $30 \%$ variation in equatorial velocity over a year. Multiple observations of the RM effect in the same hot-star system over time could provide an important test. Similarly, inclination measurements of massive stars over time should show variations.

One way to distinguish this process, in which the mis-alignment is rooted in angular momentum variations in the star alone, from one in which the planets themselves are mis-aligned, would be the observation of multiple planets in the same system with similar mis-alignments. This would be difficult to produce in a scattering-circularization scenario. Recent obser- vations of the multi-planet system Kepler-30 (SanchisOjeda et al. 2012) around a solar type star does not provide a test of our model. Observations of multi-planet systems around a hot star would provide a crucial test.

This wave-mean flow interaction could have several other interesting implications. The surface angular velocity achieved in this model is unstable to the shear instability, likely resulting in turbulence and substantial mixing of species. The angular velocities achieved here are about $2 \% \Omega_{c}$ and higher angular velocities are likely possible. These amplitudes, combined with the short timescale of angular velocity variation could explain the observed BE class of stars.

How this mechanism operates in three-dimensions (3D) is not clear. In our own atmosphere IGW driven shear flows are limited to equatorial regions because the IGW forcing is offset by the Coriolis force at higher latitudes (Baldwin et al. 2001). Although the analysis may be slightly altered in massive stars (as hydrostatic and geostrophic balance do not apply), it is highly likely these stars have latitudinal differential rotation, which should be accounted for when modeling the RM effect. More detailed analysis of latitudinal and temporal variation in surface angular velocity await more sophisticated simulations.

We suggest several observational tests of this scenario. RM observations should be analyzed assuming some latitudinal differential rotation. Repeated observations of the RM effect associated with the same hot star system would be a critical test and finally, observation of a multiple-planet system around a hot star in which all of the planets had the same mis-alignment would provide strong support for this model.

\section{REFERENCES}

Baldwin, M. P., et al. 2001, Reviews of Geophysics, 39, 179

Bate, M. R. 2010, Monthly Notices of the Royal Astronomical Society: Letters, 404, L79

Booker, J. R., \& Bretherton, F. P. 1967, Journal of Fluid Mechanics, 27, 513

Chatterjee, S., Ford, E. B., Matsumura, S., \& Rasio, F. A. 2008, The Astrophysical Journal, 686, 580

Eggleton, P. P. 1971, Monthly Notices of the Royal Astronomical Society, 151, 351, a\&AA ID. AAA005.065.032

Fabrycky, D., \& Tremaine, S. 2007, The Astrophysical Journal, 669,1298

Guillochon, J., Ramirez-Ruiz, E., \& Lin, D. 2011, The Astrophysical Journal, 732, 74

Hébrard, G., et al. 2008, Astronomy and Astrophysics, 488, 763

Hirano, T., Narita, N., Sato, B., Winn, J. N., Aoki, W., Tamura, M., Taruya, A., \& Suto, Y. 2011, Publications of the

Astronomical Society of Japan, 63, L57, (c) 2011: Astronomical Society of Japan

Howard, A. W., et al. 2010, Science, 330, 653, (c) 2010: Science

Ida, S., \& Lin, D. N. C. 2004, The Astrophysical Journal, 604, 388 - 2010, The Astrophysical Journal, 719, 810

Koenigl, A. 1991, Astrophysical Journal, 370, L39

Kozai, Y. 1962, Astronomical Journal, 67, 591

Lai, D. 2012, Monthly Notices of the Royal Astronomical Society, 423,486

Lee, M. H., \& Peale, S. J. 2002, The Astrophysical Journal, 567, 596
Lin, D. N. C., Bodenheimer, P., \& Richardson, D. C. 1996, Nature, 380, 606

Liu, S., Guillochon, J., Lin, D., \& Ramirez-Ruiz, E. 2012, The Astrophysical Journal, submitted

Nagasawa, M., \& Ida, S. 2011, The Astrophysical Journal, 742, 72

Ohta, Y., Taruya, A., \& Suto, Y. 2005, The Astrophysical Journal, 622, 1118

Plumb, R. 1977, Journal of the Atmospheric Sciences

Plumb, R., \& McEwan, A. 1978, Journal of the Atmospheric Sciences

Pollack, J. B., Hubickyj, O., Bodenheimer, P., Lissauer, J. J., Podolak, M., \& Greenzweig, Y. 1996, Icarus, 124, 62

Rasio, F. A., \& Ford, E. B. 1996, Science, 274, 954

Rogers, T. M., \& Glatzmaier, G. A. 2005, Monthly Notices of the Royal Astronomical Society, 364, 1135

Sanchis-Ojeda, R., et al. 2012, Nature, 487, 449

Schlaufman, K. C. 2010, The Astrophysical Journal, 719, 602

Triaud, A. H. M. J., et al. 2010, Astronomy and Astrophysics, 524,25

Winn, J. N., et al. 2009, The Astrophysical Journal, 700, 302

—. 2010, The Astrophysical Journal Letters, 723, L223

—. 2011, The Astrophysical Journal Letters, 741, L1

Wu, Y., \& Lithwick, Y. 2011, The Astrophysical Journal, 735, 109

Wu, Y., \& Murray, N. 2003, The Astrophysical Journal, 589, 605

We are grateful to G. Glatzmaier, K.B. MacGregor and Dan Fabrycky for helpful discussions. Support for this research was provided by a NASA grant NNG06GD44G. T. Rogers is supported by an NSF ATM Faculty Position

\footnotetext{
6 We should note that three-dimensional simulations of this sort have not been conducted and are highly computationally demand-
}

ing as they would require substantial vertical resolution in order to resolve critical layers. 


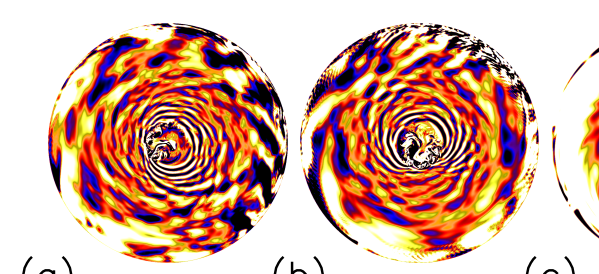

(a)

(b)

(c)

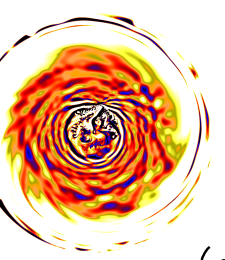

(d)

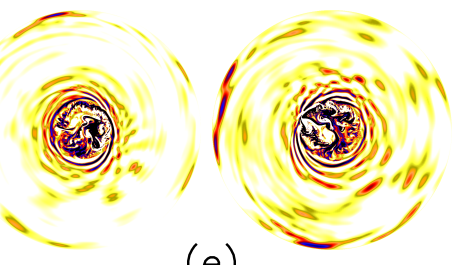

(e)

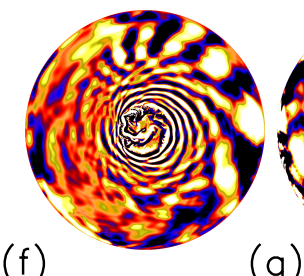

(g)

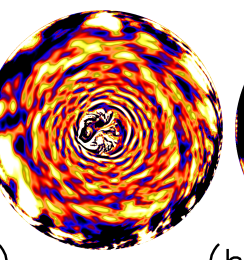

(h)

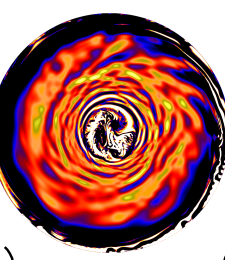

(i)

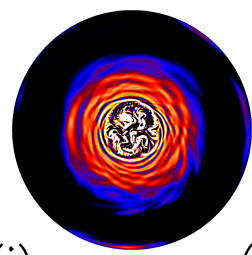

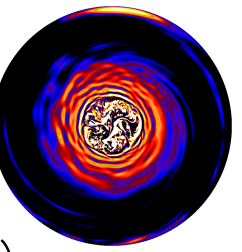

(j)

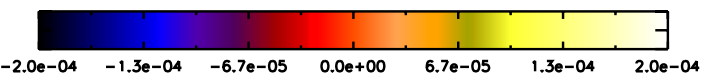

FIG. 1.- (a-e) Time snapshots of vorticity at five different times during the simulation MDR, corresponding to (a) $4.8 \times 10^{5} \mathrm{~s},(\mathrm{~b})$ $\left.1.76 \times 10^{6} \mathrm{~s}, \mathrm{c}\right) 4.6 \times 10^{6} \mathrm{~s}$, (d) $2.2 \times 10^{7} \mathrm{~s}$ and (e) $4.4 \times 10^{7} \mathrm{~s}$. (f-j) Time snapshots of vorticity at five different times during the simulation MUR, corresponding to (f) $2.57 \times 10^{6} s$, (g) $2.66 \times 10^{6} s$, (h) $5.08 \times 10^{6} s$, (i) $1.15 \times 10^{7} s$ and (j) $1.23 \times 10^{7} s$. White represents positive vorticity, while black represents negative vorticity.

in Solar physics under award number 0457631. D.N.C. Lin was supported by NASA (NNX08AM84G), NSF (AST0908807) and University of California Lab Fee. Computing resources were provided by NAS at NASA Ames. 

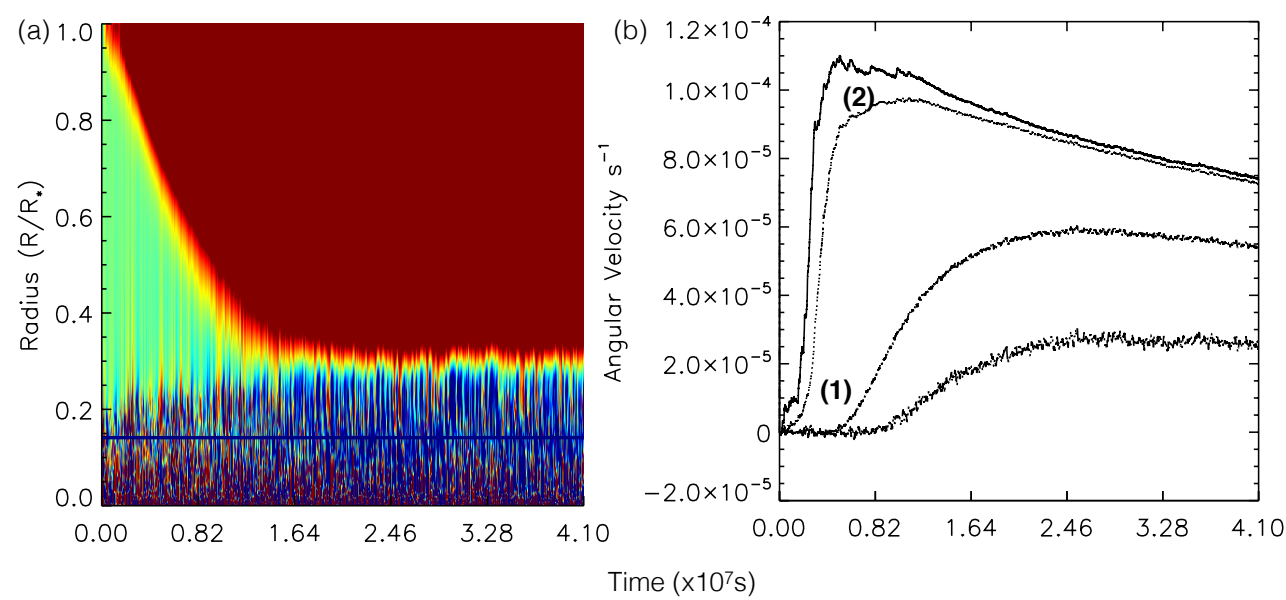

FIG. 2.- (a) Angular velocity as a function of time (x) and radius (y) for model MDR. Red represents prograde flow while blue represents retrograde flow. The blue horizontal line represents the top of the convection zone. We see a prograde layer form initially at the stellar surface and then propagate toward the convection zone in time. Initially the convection zone and overshoot layer show both prograde and retrograde flow. However, as the radiative envelope is spun up, by angular momentum conservation the convection zone must slow, as is seen. (b) Line plot of angular velocity in time for radii (moving from top to bottom) $0.98,0.86,0.45$ and 0.17 of the stellar radius. Angular momentum deposition occurs by critical level absorption between time labeled (1) and (2). The angular velocity has increased by a factor of 10 in just 9 rotation periods. Subsequent decay represents the gradual reversal process as retrograde waves now reach the surface with slightly higher amplitude. 